\title{
A NOTE ON ANALYTIC MEASURES
}

\section{NAKHLE ASMAR}

Department of Mathematics and Computer Science

California State University, Long Beach

Long Beach, California 90840

(Received March 24, 1988 and in revised form April 27, 1988)

\begin{abstract}
ABS'TRACT: Let $\mathrm{G}$ be a compact Abelian group with character group $\mathrm{X}$. Let $\mathrm{S}$ be a subset of $\mathrm{X}$ such that, for some real-valued homomorphism $\psi$ on $\mathrm{X}$, the set $\left.\left.\mathrm{S} \cap \psi^{-1}(]-\infty, \psi(\chi)\right]\right)$ is finite for all $\chi$ in $\mathrm{X}$. Suppose that $\mu$ is a measure in $\mathrm{M}(\mathrm{G})$ such that $\hat{\mu}$ vanishes off of $S$, then $\mu$ is absolutely continuous with respect to the Haar measurc on $\mathrm{G}$.
\end{abstract}

KEY WORDS AND PHRASES. Analytic measures, absolutely continuous, Bochner's Theorem.

1980 MATHEMATICS SUBJECT CLASSIFICATION CODE. 43A05 43A17

\section{INTRODUCTION.}

Let $\mathrm{G}$ denote a compact Abelian group with character group $\mathrm{X}$. Suppose that $\psi$ is a real-valued homomorphism on $\mathrm{X}$, and let $\phi$ denote the adjoint homomorphism of $\psi$. Thus $\phi$ is the continuous homomorphism from $\mathbf{R}$ into $\mathrm{G}$ such that the identity $\chi \circ \phi(r)=\exp (\imath \psi(\chi) r)$ holds for all $r$ in $\mathbf{R}$, and all $\chi$ in $\mathrm{X}$. We denote by $\mathrm{M}(\mathrm{G})$ the linear space of all complex-valued regular Borel measures on G. In the terminology of de Leew and Glicksberg [1], a measure $\mu$ in $\mathrm{M}(\mathrm{G})$ is called $\phi$-analytıc if its Fourier transform $\hat{\mu}$ vanishes on $\{\chi \in \mathrm{X}: \psi(\chi)<0\}$.

Suppose that $S$ is a nonvoid subset of $X$. Let $M_{S}(G)$ denote the closed linear subspace of $M(G)$ consisting of the measures $\mu$ with $\hat{\mu}$ vanishing off of $\mathrm{S}$. The set $\mathrm{S}$ will be called a B-set (B for Bochner) if there is a nonzero homomorphism $\psi$ from $\mathrm{X}$ into $\mathbf{R}$ such that the set $\left.\left.\mathrm{S} \cap \psi^{-1}(]-\infty, \psi(\chi)\right]\right)$ is finite for all $\chi$ in $\mathrm{X}$. The homomorphism $\psi$ may depend on S, and may not be unique. For example, a sector with opening less than $\pi$ in the lattice plane $\mathbf{Z} \times \mathbf{Z}$ is a B-set. The first orthant in $\mathbf{Z}^{\omega}$ (the weak direct product of countably many copies of $\mathbf{Z}$ ) is also a B-set. Once we have chosen a homomorphism $\psi$, we will refer to $\mathrm{S}$ as a B-set with respect to the homomorphism $\psi$.

A theorem due to Bochner [2], on $\mathbf{T}^{2}$, the two-dimensional torus, asserts that if $\mu \in M\left(\mathbf{T}^{2}\right)$ is such that $\hat{\mu}$ vanishes off of a sector of opening less than $\pi$, then $\mu$ is absolutely continuous. (The expression "absolutely continuous" will always mean absolutely continuous with respect to the Haar measure on the group in consideration.) A generalization of this result is given in de Leew and Glicksberg [1], Theorem (3.4).

It is easy to construct B-sets in $\mathbf{Z} \times \mathbf{Z}$ that are contained in no sector with opening less than $\pi$. For example, consider the set $S=\{(x, y) \in \mathbf{Z} \times \mathbf{Z}: \quad y \geq \log (1+|x|)\}$. Using results from [1], we will show that the conclusion of Bochner's theorem holds for B-sets. We have the following theorem.

(1.1) THEOREM. Let $S$ be a B-set in $X$. Suppose that $\mu$ is in $M_{S}(G)$, then $\mu$ is absolutely continuous. 
Before proving the theorem we make a few observations. Suppose that $S$ is a B-set, with respect to sonk homomorphism $\psi^{\prime}$. Clearly, there is a character $\gamma_{0}$ in $\mathrm{S}$ such that $\psi\left(x_{0}\right) \leq \psi(\gamma)$ for all $\backslash$ in $S$. Note that any translate of $S$ by an element of $X$ is also a B-set with respect to the same homomorphism $\psi$. Hence by shifting $S$ by $-\lambda_{0}$, if necessary, we may suppose that $\psi^{\prime}(\chi) \geq 0$ for all $\chi$ in $\mathrm{S}$. In this case, given a measure $\mu$ in $\mathrm{M}_{\mathrm{S}}(\mathrm{G})$, we consider the measure $\overline{\chi_{0}} \mu$ which is in $\mathrm{M}_{\mathrm{S}-\chi_{0}}(\mathrm{G})$. The set $\mathrm{S}-\chi_{0}$ is a B-set, with respect to the homomorphism $\psi ;$ and $\bar{\chi}_{0} \mu$ is absolutely continuous if and only if $\mu$ is.

If $\mu$ is in $\mathrm{M}(\mathrm{G})$, we write $\mu_{\mathrm{a}}$ and $\mu_{\mathrm{s}}$ to denote its absolutely continuous part and its singular part respectively.

(1.2) Lemma. Let $S$ be a B-set in $X$. Suppose that $\mu$ is in $M_{S}(G)$, then $\mu_{a}$ and $\mu_{S}$ are in $M_{S}(G)$.

Proof. As we observed before the lemma, we may suppose that $\psi(\mathrm{S}) \subseteq[0, \infty]$. Let $\phi$ denote the adjoint homomorphism of $\psi$, and let $\chi_{1}$ be an arbitrary character in $\mathrm{X} \backslash \mathrm{S}$, the complement of $\mathrm{S}$ in $\mathrm{X}$. We want to show that

$$
\hat{\mu}_{\mathbf{s}}\left(\chi_{1}\right)=\hat{\mu}_{\mathbf{a}}\left(\chi_{1}\right)=\mathbf{0} \text {. }
$$

First, note that if $S$ is finite then $\mu=\mu_{a}$, and the lenma is obviously true. So suppose for the rest of the proot that $\mathrm{S}$ is infinite. Let $\chi_{2}$ in $\mathrm{X}$ be such that $\psi\left(\chi_{1}\right)<\psi\left(\chi_{2}\right)$. Let $\mathrm{A}=\left\{\chi \in \mathrm{X}: \quad \psi(\chi)<\psi^{\prime}\left(\chi_{2}\right)\right\} \cap \operatorname{supp} \hat{\mu}$. The set $A$ is either void or finite. Define the measure $\sigma$ in $M(G)$ by,

$$
\sigma=\mu-\sum_{\chi \in \mathrm{A}} \dot{\mu}(\chi) \chi,
$$

where the above sum is 0 if $A$ is empty. We have

$$
\hat{\sigma}(\chi)= \begin{cases}\hat{\mu}(\chi) & \text { if } \chi \notin \mathrm{A} ; \\ 0 & \text { if } \chi \in \mathrm{A} .\end{cases}
$$

Hence $\hat{\sigma}$ vanishes off of $\psi^{-1}\left(\left[\psi\left(\chi_{2}\right), \infty[) \cap S\right.\right.$, which implies that $\sigma$ is $\phi$-analytic. It follows from [1], the Main Theorem , Proposition (2.3.2), and Theorem (5.1), that $\hat{\sigma}_{\mathbf{a}}$ and $\hat{\sigma}_{\mathbf{S}}$ vanish off of $\psi^{-1}\left(\left[\psi\left(\chi_{2}\right), \infty[) \cap \mathrm{S}\right.\right.$. Since $\mu_{\mathrm{S}}=\sigma_{\mathrm{S}}$, it follows that $\hat{\mu}_{\mathrm{S}}$ vanishes off of $\psi^{-1}\left(\left[\psi\left(\chi_{2}\right), \infty[) \cap \mathrm{S}\right.\right.$. Therefore, $\hat{\mu}_{\mathrm{s}}\left(\chi_{1}\right)=0$, and the lemma follows.

Proof of Theorem (1.1). According to Lemma (1.2), it is enough to show that $\hat{\mu}_{\mathrm{S}}(\chi)=0$ for all $\chi$ in $\mathrm{S}$. The proof is by contradiction. Assume that $\hat{\mu}_{\mathrm{S}}\left(\chi_{0}\right) \neq 0$ for some $\chi_{0}$ in S. Let $\chi_{1}$ in $\mathrm{X}$ be such that $\psi\left(\chi_{1}\right)>\psi\left(\chi_{0}\right)$. (Here also we are assuming that $S$ is infinite and $\psi(S) \subseteq\left[0, \infty[\right.$.$) Let \mathbf{A}=\left\{\chi \in X: \quad \psi(\chi) \leq \psi\left(\chi_{1}\right)\right.$, and $\left.\hat{\mu}_{\mathrm{s}}(\chi) \neq 0\right\}$. Then $\mathrm{A}$ is contained in $\left.\left.\psi^{-1}(]-\infty, \psi\left(\chi_{1}\right)\right]\right) \cap \mathrm{S}$; and so $\mathrm{A}$ is finite and $\chi_{0}$ is in $\mathrm{A}$. Define the measure $\nu$ in $\mathrm{M}(\mathrm{G})$ by

We have

$$
\nu=\mu_{\mathrm{s}}-\sum_{\chi \in \mathrm{A}} \hat{\mu}_{\mathrm{s}}(\chi) \chi
$$

$$
\hat{\nu}(\chi)= \begin{cases}\hat{\mu}_{\mathrm{s}}(\chi) & \text { if } \chi \notin \mathrm{A} ; \\ 0 & \text { if } \chi \in \mathrm{A} .\end{cases}
$$

Thus $\hat{\nu}$ vanishes off of $\psi^{-1}\left(\left[\psi\left(\chi_{1}\right), \infty[) \cap S\right.\right.$, and hence it is $\phi$-analytic. Applying Proposition (5.1), [1], we see that $\hat{\nu}_{\mathrm{S}}$ and $\hat{\nu}_{\mathrm{a}}$ vanish off of $\psi^{-1}\left(\left[\psi\left(\chi_{1}\right), \infty[) \cap \mathrm{S}\right.\right.$. Since $\nu_{\mathrm{s}}=\mu_{\mathrm{S}}$, it follows that $\hat{\mu}_{\mathrm{s}}$ vanishes off of $\psi^{-1}\left(\left[\psi\left(\chi_{1}\right), \infty[) \cap \mathrm{S}\right.\right.$. This is plainly a contradiction since $\psi\left(\chi_{0}\right)<\psi\left(\chi_{1}\right)$, and by assumption $\hat{\mu}_{\mathrm{s}}\left(\chi_{0}\right) \neq 0$.

\section{REFERENCES}

1. de Leew, K., and I. Glicksberg. Quasi-Invariance and analyticity of measures on compact groups. Acta. Math. 103 1963, 179-205

2. Bochner, S. Boundary values of analytic functions in several variables and almost periodic functions. Ann. of Math. 15 1944, 708-7:22 


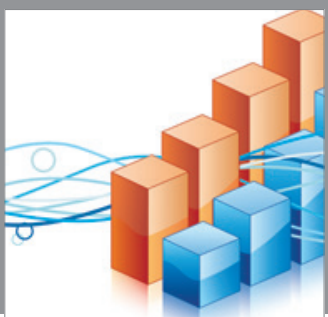

Advances in

Operations Research

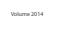

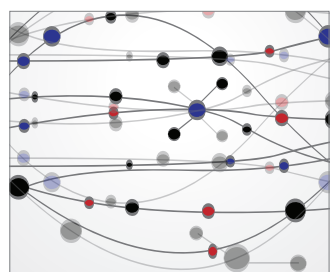

\section{The Scientific} World Journal
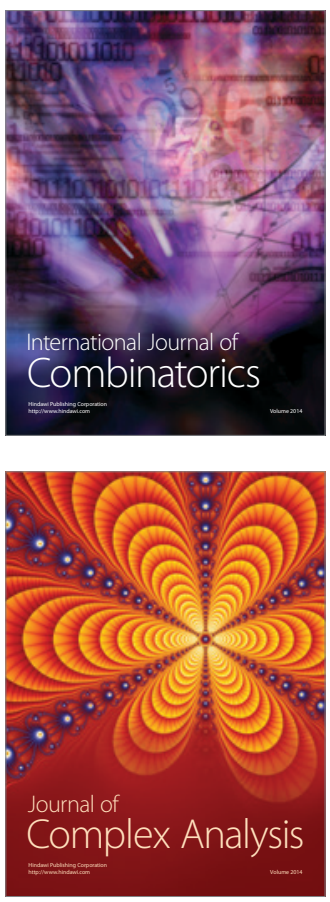

International Journal of

Mathematics and

Mathematical

Sciences
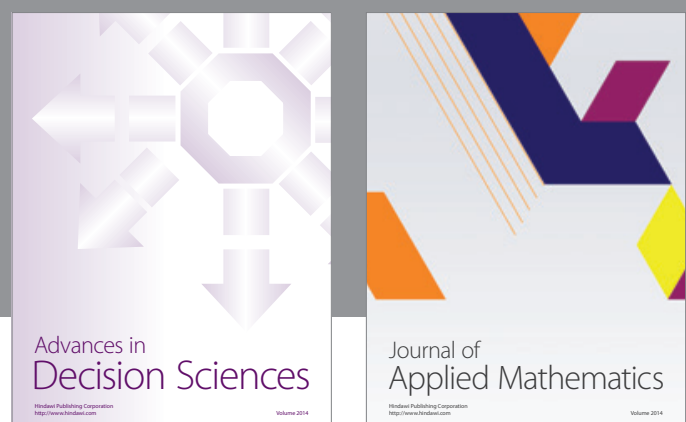

Journal of

Applied Mathematics
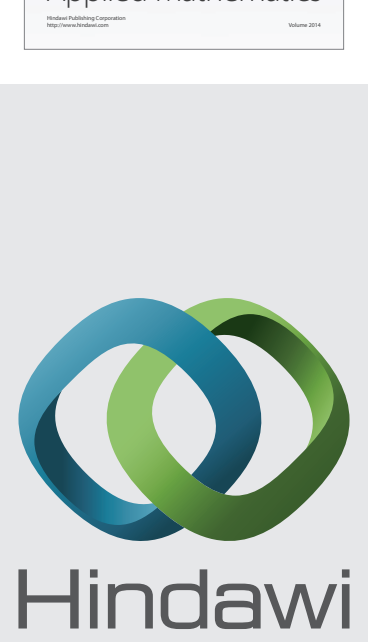

Submit your manuscripts at http://www.hindawi.com
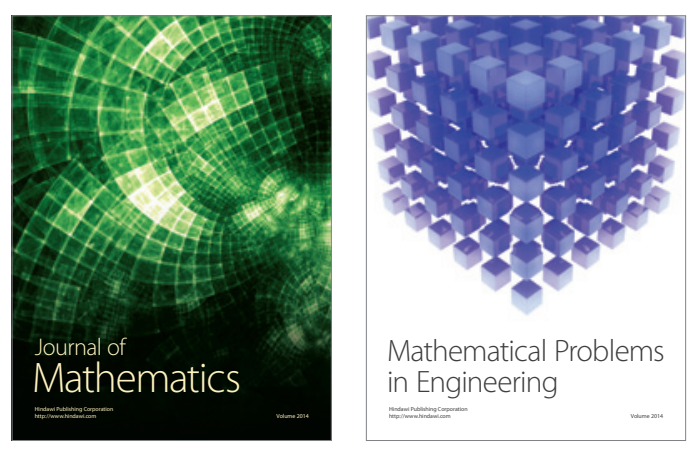

Mathematical Problems in Engineering
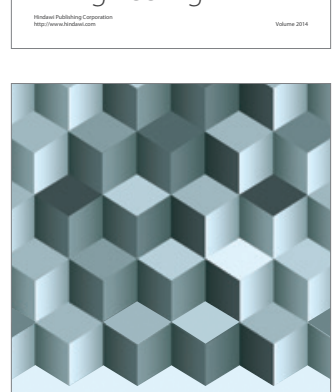

Journal of

Function Spaces
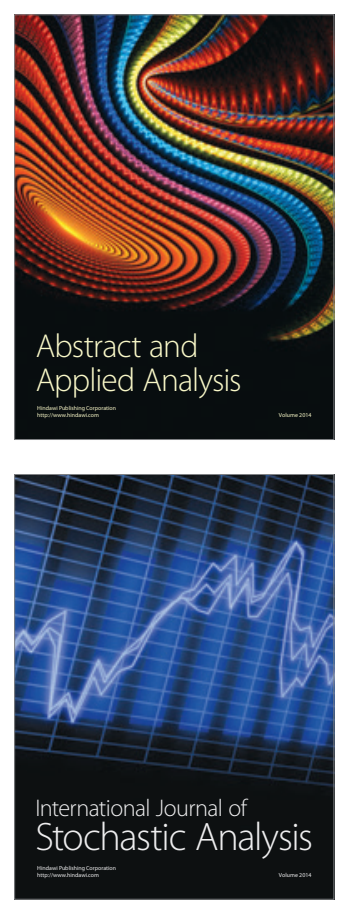

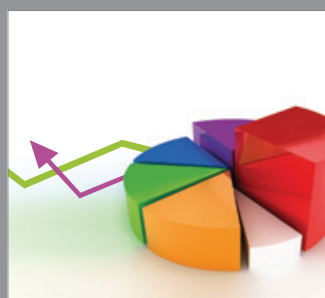

ournal of

Probability and Statistics

Promensencen
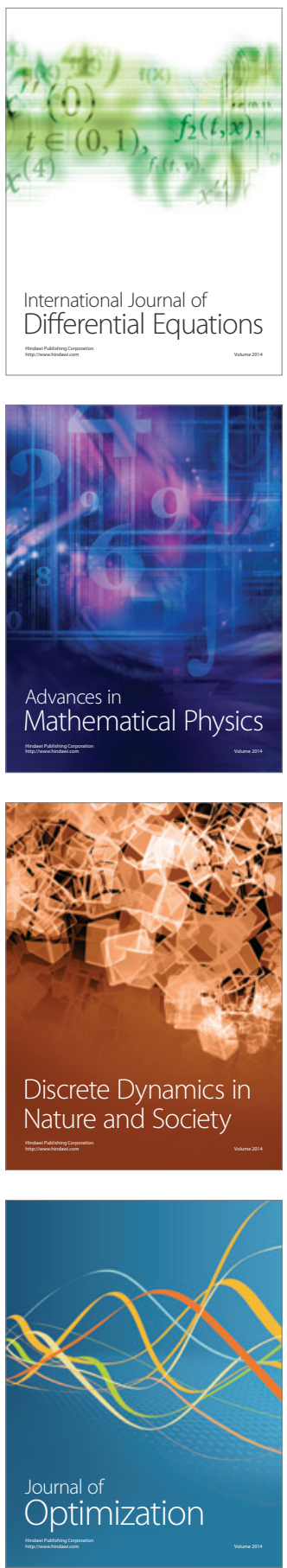